\title{
AUTHOR INDEX Volume 24
}

A. Größlinger and Köstler, H., Guest editors' note: Special issue on high-performance stencil computations

24 (2014) 1402002

Abdelhamid, N., Ayesh, A. and Hadi, W., Multi-label rules algorithm based associative classification

24 (2014) 1450001

Akl, S. G., Editorial note

24 (2014) 1401001

Akl, S. G., Editorial note

24 (2014) 1401002

Akl, S. G., Editorial note

24 (2014) 1401003

Akl, S. G., Editorial note

24 (2014) 1401004

Alkowaileet, W. Y., Carrillo-Cisneros, D., Lim, R. V. and

Scherson, I. D., NUMA-Aware multicore matrix multiplication

24 (2014) 1450006

Apel, S., see Grebhahn, A.

Ayesh, A., see Abdelhamid, N.

24 (2014) 1441001

Bader, M., see Weinzierl, T.

24 (2014) 1450001

24 (2014) 1441006

Bettencourt, M., see Lin, P.

24 (2014) 1442005

Breuer, S., see Steuwer, M.

24 (2014) 1441005

Cameron, K., see Li, B.

Carrillo-Cisneros, D., see Alkowaileet, W. Y.

24 (2014) 1442001

24 (2014) 1450006

Chang, H.-C., see Li, B.

24 (2014) 1442001

24 (2014) 1442002

Chen, Y., see Leidel, J. D.

24 (2014) 1441002

Dediu, A.-H., Martín-Vide and Truthe, B., Guest editors' note: Theory and practice of natural computing

Domino, S., see Lin, P.

24 (2014) 1402001

24 (2014) 1442005

Dongarra, J., see Yamazaki, I.

24 (2014) 1442004

Feo, J., see Lucas, A.

Fisher, T., see Lin, P.

Freivalds, R., see Kucevalovs, I.

24 (2014) 1450004

24 (2014) 1442005

24 (2014) 1440003

Fuhrer, O., see Lapillonne, X.

24 (2014) 1450003

24 (2014) 1440001

Gioios, R Kestor, G. and Kerbyson, D. J Ond applications

24 (2014) 1442003 systems for performance fault detection

24 (2014) 1441005

Gorlatch, S., see Steuwer, M.

Grebhahn, A., Kuckuk, S., Schmitt, C., Köstler, H., Siegmund, N., Apel, S., Hannig, F. and Teich, J., Experiments on 
optimizing the performance of stencil codes with SPL conqueror

Grosser, T., Verdoolaege, S., Cohen, A. and Sadayappan, P.,

The relation between diamond tiling and hexagonal tiling

Hack, S., see Köster, M.

Hadi, W., see Abdelhamid, N.

Hager, G., see Kronawitter, S.

Haidl, M., see Steuwer, M.

Hannig, F., see Grebhahn, A.

Hoemmen, M., see Lin, P.

Holloway, D. M., see Spirov, A. V.

$\mathrm{Hu}$, J., see Lin, P.

Kakugawa, H., see Kawai, S.

Kawai, S., Ooshita, F., Kakugawa, H. and Masuzawa, T., Analysis of distributed token circulation algorithm with faulty random number generator

Kennon, S., see Lin, P.

Kerbyson, D. J., see Gioiosa, R.

Kerbyson, D. J., see Weems, C. C.

Kestor, G., see Gioiosa, R.

Kmet, T., Neural networks solving free and fixed final time optimal control problems

Köster, M., R. LeißaHack, S., Membarth, R. and Slusallek, P., Code refinement of stencil codes

Köstler, H., see Grebhahn, A.

Köstler, H., see Größlinger, A.

Krasts, O., see Kucevalovs, I.

Kronawitter, S., Stengel, H., Hager, G. and Lengauer, C., Domain-specific optimization of two Jacobi smoother kernels and their evaluation in the ECM performance model

24 (2014) 1441002

24 (2014) 1441003

24 (2014) 1450001

24 (2014) 1441004

24 (2014) 1441005

24 (2014) 1441001

24 (2014) 1442005

24 (2014) 1440004

24 (2014) 1442005

24 (2014) 1450002

24 (2014) 1450002

24 (2014) 1442005

24 (2014) 1442003

24 (2014) 1402003

24 (2014) 1442003

24 (2014) 1440002

24 (2014) 1441003

24 (2014) 1441001

24 (2014) 1402002

24 (2014) 1440003

24 (2014) 1441004

Kucevalovs, I., Krasts, O., Freivalds, R. and Zeugmann, T., On the influence of technology on learning processes

Kuckuk, S., see Grebhahn, A.

Kurzak, J., see Yamazaki, I.

Lapillonne, X. and Fuhrer, O., Using compiler directives to port large scientific applications to GPUs: An example from atmospheric science

24 (2014) 1440003

24 (2014) 1441001

24 (2014) 1442004

24 (2014) 1450003

Leidel, J. D. and Chen, Y., HMC-SIM: A simulation framework for hybrid memory cube devices

24 (2014) 1442002

24 (2014) 1441004

Li, B., Chang, H.-C., Song, S., Su, C.-Y., Meyer, T., Mooring, J. and Cameron, K., Extending PowerPack for profiling and analysis of high-performance accelerator-based systems

Lim, R. V., see Alkowaileet, W. Y.

24 (2014) 1442001

24 (2014) 1450006

Lin, P., Bettencourt, M., Domino, S., Fisher, T., Hoemmen, M., Hu, J., Phipps, E., Prokopenko, A., Rajamanickam, S., 
Siefert, C. and Kennon, S., Towards extreme-scale simulations for low mach fluids with second-generation trilinos

24 (2014) 1442005

Lucas, A., Stalzer, M. and Feo, J., Parallel implementation of fast randomized algorithms for low rank matrix decomposition Luszczek, P., see Yamazaki, I.

Martín-Vide, see Dediu, A.-H.

Masuzawa, T., see Kawai, S.

Membarth, R., see Köster, M.

Meyer, T., see Li, B.

Mooring, J., see Li, B.

Ooshita, F., see Kawai, S.

Phipps, E., see Lin, P.

Prokopenko, A., see Lin, P.

R. Leißa, see Köster, M.

Rajamanickam, S., see Lin, P.

Rajamony, R., see Weems, C. C.

Sadayappan, P., see Grosser, T.

Scherson, I. D., see Alkowaileet, W. Y.

Schmitt, C., see Grebhahn, A.

Siebert, C. and Träff, J. L., Perfectly load-balanced, stable, synchronization-free parallel merge

Siefert, C., see Lin, P.

Siegmund, N., see Grebhahn, A.

Slusallek, P., see Köster, M.

Song, S., see Li, B.

Spirov, A. V., Zagriychuk, E. A. and Holloway, D. M., Evolutionary design of gene networks: Forced evolution by genomic parasites

Stalzer, M., see Lucas, A.

Stengel, H., see Kronawitter, S.

Steuwer, M., Haidl, M., Breuer, S. and Gorlatch, S., High-level programming of stencil computations on multi-GPU systems using the skelcl library

$\mathrm{Su}, \mathrm{C} .-\mathrm{Y} .$, see $\mathrm{Li}, \mathrm{B}$.

Teich, J., see Grebhahn, A.

Träff, J. L., see Siebert, C.

Truthe, B., see Dediu, A.-H.

Unterweger, K., see Weinzierl, T.

Verdoolaege, S., see Grosser, T.

Weems, C. C., Kerbyson, D. J. and Rajamony, R., Guest editors' note: Special issue on large-scale parallel processing

24 (2014) 1450004

24 (2014) 1442004

24 (2014) 1402001

24 (2014) 1450002

24 (2014) 1441003

24 (2014) 1442001

24 (2014) 1442001

24 (2014) 1450002

24 (2014) 1442005

24 (2014) 1442005

24 (2014) 1441003

24 (2014) 1442005

24 (2014) 1402003

24 (2014) 1441002

24 (2014) 1450006

24 (2014) 1441001

24 (2014) 1450005

24 (2014) 1442005

24 (2014) 1441001

24 (2014) 1441003

24 (2014) 1442001

24 (2014) 1440004

24 (2014) 1450004

24 (2014) 1441004

24 (2014) 1441005

24 (2014) 1442001

24 (2014) 1441001

24 (2014) 1450005

24 (2014) 1402001

24 (2014) 1441006

24 (2014) 1441002

Weinzierl, T., Bader, M., Unterweger, K. and Wittmann, R., Block fusion on dynamically adaptive spacetree grids for shallow water waves

24 (2014) 1402003

Wittmann, R., see Weinzierl, T.

24 (2014) 1441006

24 (2014) 1441006 
Yamazaki, I., Kurzak, J., Luszczek, P. and Dongarra, J., Design and implementation of a large scale tree-based QR decomposition using a $3 \mathrm{D}$ virtual systolic array and a lightweight runtime

24 (2014) 1442004

Zagriychuk, E. A., see Spirov, A. V.

24 (2014) 1440004

Zeugmann, T., see Kucevalovs, I.

24 (2014) 1440003 